A paper to be presented at the Annual Conven-

tion Meeting of the A mercian Institute of Elec-

trical Engineers, Niagara Falls, June 28, 1907

Copyright 1907 By A I.E.E.

(Subject to final revision for the Transactions.)

\title{
INDUCTIVE DISTURBANCES IN TELEPHONE LINE
}

BY LOUIS COHEN.

The inductive action of one telephone line on another, producing what is commonly known as cross-talk, presents an important problem to the telephone engineer. The induction is both electromagnetic and electrostatic. A variable current passing through any circuit is accompanied by a variable magnetic field, which will produce electric currents in neighboring circuits. In addition to this there exists also an electrostatic effect, a charge on one conductor will induce charges on all neighboring conductors, and any variation in the charge will produce an electric current. We thus have two distinct phenomena acting simultaneously, both causing trouble on telephone lines. It is to be noticed, however, that the two effects act in opposite directions. The establishment of a current in one circuit is accompanied by an induced current in any neighboring circuit in the opposite direction, while when the current in the inducing circuit is decreasing, the induced current will be in the same direction. In the case of electrostatic induction the conditions are reversed. When both forms of induction act together we obtain a resultant effect which depends on the various electrical constants of the lines and their relative importance. It would appear therefore quite evident that to obtain a knowledge of the effect due to induction, we must first be able to ascertain the relative importance of one form of induction as compared with the other, and to determine the factors which enter in fixing the magnitude of each. A thorough knowledge of these factors may be of considerable assistance in improving the conditions of telephone lines, or at least it 
may suggest some methods for overcoming induction effects which are a source of considerable annoyance to users of telephones.

In a paper presented before the American Institute of Electrical Engineers in 1891,* Mr. J. J. Carty discussed a series of experiments which he had conducted to determine the relative importance of the electrostatic as compared with the electromagnetic induction. His conclusions were that the electromagnetic effect is entirely negligible as compared with the electrostatic effect; in fact in his experiments he has not been able to detect any electromagnetic effect at all. In the discussion of his paper Mr. Carty made the following statement:

"I go so far as to set forth that the effect of electromagnetic induction between parallel telephone wires may be neglected. That is, that when a man is talking on one wire and his speech is heard by induction on a parallel wire, that that speech finds its way between the two wires

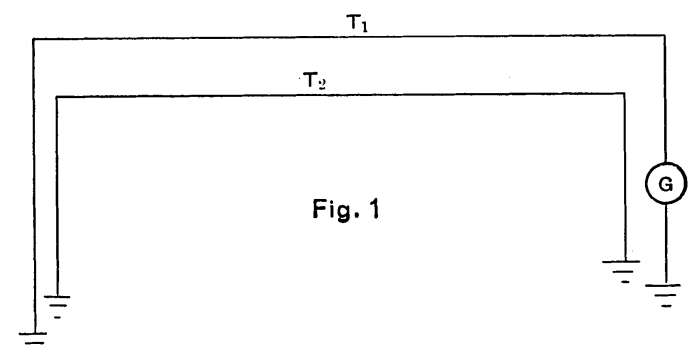

by virtue of electrostatic induction, and that electromagnetic induction is entirely negligible."

This view expressed by $\mathrm{Mr}$. Carty has generally been accepted by telephone engineers, and is usually given a very prominent place in all text books on telephony.

In what follows I shall discuss this question from a mathematical standpoint, and shall show that not only is the electromagnetic induction not a negligible quantity, but that in some cases the electromagnetic effect may be much larger than the electrostatic effect; and I shall further show that the results Mr. Carty obtained were correct, that under the conditions of Mr. Carty's experiments he could have obtained only an electrostatic effect. To infer, however, from his results, as Mr. Carty has done, that what is true for the case he experimented with-

* J. J. Carty, Transactions. of the A. I. E. E. 1891. Vol. 8, page 114. 
a line three hundred feet long-is equally true for a line so many miles long is, as I will show, quite erroneous.

Suppose we have two lines $T_{1}$ and $T_{2}$ running parallel to each other and both are grounded at the two ends; $T_{1}$ may be a power circuit or telephone circuit, $G$ is a source of an alternating electromotive force, and $T_{2}$ a telephone line. We wish to consider what is the nature of the induced currents in line $T_{2}$.

Let $L_{1}, R_{1}, C_{1}$, denote the self-inductance, resistance, and capacity per unit length of line $T_{1}$, and similar letters with suffix 2 denote the same constants of line $T_{2} ; M$ will denote the mutual electromagnetic inductance and $C_{12}$ the mutual electrostatic inductance. Let also $x$ and $y$ denote the currents, $V_{1}$ and $V_{2}$ the potentials at any point on the lines $T_{1}$ and $T_{2}$.

The algebraic sum of the forces acting at any point on the two lines will be given by the following equations:

$$
\begin{aligned}
& L_{1} \frac{d x}{d t}+R_{1} x+M \frac{d y}{d t}+\frac{d V_{1}}{d s}=0 \\
& L_{2} \frac{d y}{d t}+R_{2} y+M \frac{d x}{d t}+\frac{d V_{2}}{d s}=0
\end{aligned}
$$

We also have the electrostatic relation,

$$
\begin{aligned}
& C_{1} V_{1}+C_{12} V_{2}=q_{1} \\
& C_{2} V_{2}+C_{12} V_{1}=q_{2}
\end{aligned}
$$

By the aid of the equations of continuity

$$
\begin{aligned}
& \frac{d q_{1}}{d t}=-\frac{d x}{d s} \\
& \frac{d q_{2}}{d t}=-\frac{d y}{d s}
\end{aligned}
$$

we may write equations (2) after differentiating with respect to $t$ in the following form:

$$
\begin{gathered}
C_{1} \frac{d V_{1}}{d t}+C_{12} \frac{d V_{2}}{d t}=-\frac{d x}{d s} \\
C_{2} \frac{d V_{2}}{d t}+C_{12} \frac{d V_{1}}{d t}=-\frac{d y}{d s}
\end{gathered}
$$


Differentiating equations (1) with respect to $s$ we get:

$$
\begin{aligned}
& L_{1} \frac{d^{2} x}{d t d s}+R_{1} \frac{d x}{d s}+M \frac{d^{2} y}{d t d s}+\frac{d^{2} V_{1}}{d s^{2}}=0 \\
& L_{2} \frac{d^{2} y}{d t d s}+R_{2} \frac{d y}{d s}+M \frac{d^{2} x}{d t d s}+\frac{d^{2} V_{2}}{d s^{2}}=0
\end{aligned}
$$

If we differentiate equations (3) with respect to $t$, and introduce the values of $\frac{d^{2} x}{d s d t}, \frac{d^{2} y}{d s d t}$ thus obtained into equations (4) and also the values of $\frac{d x}{d s}$ and $\frac{d y}{d s}$ from equations (3) we shall obtain the following equations:

$$
\begin{gathered}
L_{1} C_{1} \frac{d^{2} V_{1}}{d t^{2}}+L_{1} C_{12} \frac{d^{2} V_{2}}{d t^{2}}+R_{1} C_{1} \frac{d V_{1}}{d t}+R_{1} C_{12} \frac{d V_{2}}{d t} \\
+M C_{2} \frac{d^{2} V_{2}}{d t^{2}}+M C_{12} \frac{d^{2} V_{1}}{d t^{2}}=\frac{d^{2} V_{1}}{d s^{2}} \\
L_{2} C_{2} \frac{d^{2} V_{2}}{d t^{2}}+L_{2} C_{12} \frac{d^{2} V_{1}}{d t^{2}}+R_{2} C_{2} \frac{d V_{2}}{d t}+R_{2} C_{12} \frac{d V_{1}}{d t} \\
+M C_{1} \frac{d^{2} V_{1}}{d t}+M C_{12} \frac{d^{2} V_{2}}{d t^{2}}=\frac{d^{2} V_{2}}{d s^{2}}
\end{gathered}
$$

Assuming that the impressed electromotive force be simple harmonic, that is the real part of $E e^{i p t}$ say, then the potentials at any point along the lines will be simple harmonic, and therefore we may put:

$$
\begin{aligned}
& \frac{d^{2} V_{1}}{d t^{2}}=-p^{2} V_{1}, \quad \frac{d V_{1}}{d t}=i p V_{1} \\
& \frac{d^{2} V_{2}}{d t^{2}}=-p^{2} V_{2}, \quad \frac{d V_{2}}{d t}=\imath p V_{2}
\end{aligned}
$$


Introducing these values in equation (5) and rearranging we get:

$$
\begin{aligned}
& \frac{d^{2} V_{1}}{d s^{2}}=a_{1} V_{1}+b_{1} V_{2} \\
& \frac{d^{2} V_{2}}{d s^{2}}=a_{2} V_{2}+b_{2} V_{1}
\end{aligned}
$$

Where,

$$
\begin{aligned}
& a_{1}=-p^{2} L_{1} C_{1}+i p R_{1} C_{1}-M C_{12} p^{2} \\
& b_{1}=-p^{2} L_{1} C_{12}+i p R_{1} C_{12}-M C_{2} p^{2} \\
& a_{2}=-p^{2} L_{2} C_{2}+i p R_{2} C_{2}-M C_{12} p^{2} \\
& b_{2}=-p^{2} L_{2} C_{12}+i p R_{2} C_{12}-M C_{1} p^{2}
\end{aligned}
$$

Each of the equations (6) contains two dependent variables which make it rather difficult to solve. We can, however, transform equations (6) into equations containing only one dependent variable by the following device:*

Put

$$
\begin{aligned}
& V_{1}=W_{1}+W_{2} \\
& V_{2}=f_{1} W_{1}+f_{2} W_{2}
\end{aligned}
$$

where $f_{1}$ and $f_{2}$ are arbitrary constants.

Equations (6) will transform into the following:

$$
\begin{array}{r}
\frac{d^{2} W_{1}}{d s^{2}}+\frac{d^{2} W_{2}}{d s^{2}}=\left(a_{1}+b_{1} f_{1}\right) W_{1}+\left(a_{1}+b_{1} f_{2}\right) W_{2} \\
f_{1} \frac{d^{2} W_{1}}{d s^{2}}+f_{2} \frac{d^{2} W_{2}}{d s^{2}}=\left(a_{2} f_{1}+b_{1}\right) W_{1}+\left(a_{2} f_{2}+b_{2}\right) W_{2}
\end{array}
$$

Eliminating first $W_{1}$ and then $W_{2}$ we get the following equations:

$$
\begin{aligned}
& \left(a_{2} f_{2}+b_{2}-a_{1} f_{1}-b_{1} f_{1} f_{2}\right) \frac{d^{2} W_{1}}{d s^{2}}+\left(a_{2} f_{2}+b_{2}-a_{1} f_{2}-b_{1} f_{2}^{2}\right) \frac{d^{2} W_{2}}{d s^{2}} \\
& =\left(a_{1} a_{2}-b_{1} b_{2}\right)\left(f_{2}-f_{1}\right) W_{1} \\
& \left(a_{2} f_{1}+C_{2}-a_{1} f_{1}-C_{1} f_{1}^{2}\right) \frac{d^{2} W_{1}}{d s^{2}}+\left(a_{2} f_{1}+C_{1}-a_{1} f_{2}-C_{1} f_{1} f_{2}\right) \frac{d^{2} W_{2}}{d s^{2}} \\
& =\left(a_{1} a_{2}-C_{1} C_{2}\right)\left(f_{1}-f_{2}\right) \mathrm{W}_{2}
\end{aligned}
$$

*See O. Heaviside coll. papers, v(.1. 1, p. 126. 
now determine $f_{2}$ and $f_{1}$ from the equations

that is,

$$
\begin{aligned}
& a_{2} f_{2}+b_{2}-a_{1} f_{2}-b_{1} f_{2}^{2}=0 \\
& a_{2} f_{1}+b_{2}-a_{1} f_{1}-b_{1} f_{1}^{2}=0
\end{aligned}
$$

$$
\begin{aligned}
& f_{2}=\frac{a_{2}-a_{1}-\sqrt{\left(a_{1}-a_{2}\right)^{2}+4 b_{1} b_{2}}}{2 b_{1}} \\
& f_{1}=\frac{a_{2}-a_{1}-\sqrt{\left(a_{1}-a_{2}\right)^{2}+4 b_{1} b_{2}}}{2 b_{1}}
\end{aligned}
$$

Equations (8) will reduce to the following:

$$
\begin{aligned}
& \frac{d^{2} W_{1}}{d s^{2}}=\overline{K^{2}} W_{1} \\
& \frac{d^{2} W_{2}}{d s^{2}}=P^{2} W_{2}
\end{aligned}
$$

where,

$$
\begin{aligned}
& \overline{K^{2}}=\frac{\left(a_{1} a_{2}-b_{1} b_{2}\right)\left(f_{2}-f_{1}\right)}{a_{2} f_{2}+b_{2}-a_{1} f_{1}-b_{1} f_{1} f_{2}} \\
& P^{2}=\frac{\left(a_{1} a_{2}-b_{1} b_{2}\right)\left(f_{1}-f_{2}\right)}{a_{2} f_{1}+b_{2}-a_{1} f_{2}-b_{1} f_{1} f_{2}}
\end{aligned}
$$

The complete solutions of (10) will be the following:

$$
\begin{aligned}
& W_{1}=A \cos \mu(l-s)+B \sin \mu(l-s) \\
& W_{2}=D \cos \mu_{1}(l-s)+F \sin \mu_{1}(l-s)
\end{aligned}
$$

The constants are to be determined from the following boundary conditions:

When

$$
\begin{array}{lll}
s=0, & V_{1}=E e^{i p t} & V_{2}=0 \\
s=l, & V_{1}=0, & V_{2}=0
\end{array}
$$

Now,

$$
W_{1}=\frac{f_{1} V_{1}-V_{2}}{f_{1}-f_{2}}, \quad W_{2}=\frac{f_{2} V_{1}-V_{2}}{f_{2}-f_{1}}
$$


Hence, when $s=0$

$$
\begin{aligned}
& W_{1}=A \cos \mu l+B \sin \mu l=\frac{f_{1} E e^{i p t}}{f_{1}-f_{2}} \\
& W_{2}=D \cos \mu_{1} l+F \sin \mu_{1} l=\frac{f_{2} E e^{i p t}}{f_{2}-f_{1}}
\end{aligned}
$$

When $s=l$,

$$
\begin{aligned}
& W_{1}=A=0 \\
& W_{2}=D=0
\end{aligned}
$$

and therefore,

$$
\begin{aligned}
& B=\frac{f_{1} E e^{i p t}}{\left(f_{1}-f_{2}\right) \sin \mu l} \\
& F=\frac{f_{2} E e^{i p t}}{\left(f_{2}-f_{1}\right) \sin \mu_{1} l}
\end{aligned}
$$

Introducing the values of the constants thus obtained into equation (12) we shall obtain the following values for $V_{1}$ and $V_{2}$ :

$$
\begin{aligned}
& V_{1}=W_{1}+W_{2}=\left(\frac{f_{1} \sin \mu(l-s)}{\sin \mu l}-f_{2} \frac{\sin \mu_{1}(l-s)}{\sin \mu_{1} l}\right) \frac{E e^{i p t}}{f_{1}-f_{2}} \\
& V_{2}=f_{1} W_{1}+f_{2} W_{2}=\left(\frac{f_{1}^{2} \sin \mu(l-s)}{\sin \mu l}-f_{2}{ }^{2} \frac{\sin \mu_{1}(l-s)}{\sin \mu_{1} l}\right) \frac{E e^{i p t}}{f_{1}-f_{2}}
\end{aligned}
$$

The values of $\mu$ and $\mu_{1}$ may be obtained by inspection of equation (10) which gives

$$
\begin{aligned}
& -\mu^{2}=\overline{K^{2}}=\frac{\left(a_{1} a_{2}-b_{1} b_{2}\right)\left(f_{2}-f_{1}\right)}{a_{2} f_{2}+b_{2}-a_{1} f_{1}-b_{1} f_{1} f_{2}} \\
& -\mu_{1}^{2}=P^{2}=\frac{\left(a_{1} a_{2}-b_{1} b_{2}\right)\left(f_{1}-f_{2}\right)}{a_{2} f_{1}+b_{1}-a_{1} f_{2}-b_{1} f_{1} f_{2}}
\end{aligned}
$$

The value of the currents in the two lines can be easily obtained from equation (13) by the aid of equation (3). Thus: 


$$
\begin{gathered}
-x=\left[C_{1} i p\left(\frac{f_{1} \cos \mu(l-s)}{\mu \sin \mu l}-f_{2} \frac{f_{2} \cos \mu_{1}(l-s)}{\mu_{1} \sin \mu_{1} l}\right)\right. \\
\left.+i C_{12} p\left(\frac{f_{1}{ }^{2} \cos \mu(l-s)}{\mu \sin \mu l}-\frac{f_{2}{ }_{2} \cos \mu_{1}(l-s)}{\mu_{1} \sin \mu_{1} l}\right)\right] \frac{E e^{i p t}}{f_{1}-f_{2}} \\
-y=\left[C_{2} i p\left(\frac{f_{1}{ }^{2} \cos \mu(l-s)}{\mu \sin \mu l}-\frac{f_{2}{ }^{2} \cos \mu_{1}(l-s)}{\mu_{1} \sin \mu_{1} l}\right)\right. \\
\left.+i C_{12} p\left(\frac{f_{1} \cos \mu(l-s)}{\mu \sin \mu l}-\frac{f_{2} \cos \mu_{1}(l-s)}{\mu_{1} \sin \mu_{1} l}\right)\right] \frac{E e^{i p t}}{f_{1}-f_{2}}
\end{gathered}
$$

Rearranging we obtain:

$$
\begin{gathered}
-x=\left(\left(C_{1} f_{1}+C_{12} \dot{f}_{1}^{2}\right) \frac{\cos \mu(l-s)}{\mu \sin \mu l}\right. \\
\left.-\left(C_{1} f_{2}+C_{12} f_{2}^{2}\right) \frac{\cos \mu_{1}(l-s)}{\mu_{1} \sin \mu_{1} l}\right) \frac{i p E e^{i p t}}{f_{1}-f_{2}} \\
-y=\left(\left(C_{2} f_{1}^{2}+C_{12} f_{1}\right) \frac{\cos \mu(l-s)}{\mu \sin \mu l}\right. \\
\left.-\left(C_{2} f_{2}^{2}+C_{12} f_{2}\right) \frac{\cos \mu_{1}(l-s)}{\mu_{1} \sin \mu_{1} l}\right) \frac{i p E e^{i p t}}{f_{1}-f_{2}}
\end{gathered}
$$

The second equation of (16) gives us the final expression for the currents in any wire due to electromagnetic and electrostatic induction of a parallel wire. The inducing line may be part of a power circuit, lighting circuit, or a similar telephone line. In deducing equations (16) we have taken account of the difference in dimensions, or electrical constants, of the two lines which react upon each other, which will of course be the case if we wish to calculate the currents induced in a telephone line by a power circuit running parallel to it.

According to some recent reports, the introduction of the single-phase electric railway plays havoc with service on telephone and telegraph lines which parallel the railways. This will in all probability be a very serious problem, and a careful consideration of equation (16) will give us a better insight into the nature of the problem and the magnitude of the disturbance. It may also possibly suggest some means for eliminating 
these disturbances or at least reducing them to a minimum. I hope to consider this phase of the problem in another paper, but for the present I shall limit my discussion to the case of two parallel telephone lines.

If it is the influence of two telephone lines on each other that we wish to investigate, then equations (16) will be somewhat simplified; for in that case we find by examining equations (6) that

$$
a_{1}=a_{2} \quad b_{1}=b_{2}
$$

equations (9) will therefore reduce to

$$
f_{1}=-f_{2}=-1
$$

Hence equations (16) will become,

$-x=\frac{1}{2}\left(\left(C+C_{12}\right) \frac{\cos \mu(l-s)}{\mu \sin \mu l}+\left(C-C_{12}\right) \frac{\cos \mu_{1}(l-s)}{\mu_{1} \sin \mu_{1} l}\right) i p E e^{i p t}$

$-y=\frac{1}{2}\left(\left(C+C_{12}\right) \frac{\cos \mu(l-s)}{\mu \sin \mu l}-\left(C-C_{12}\right) \frac{\cos \mu_{1}(l-s)}{\mu_{1} \sin \mu_{1} l}\right) \imath p E e^{i p t}$

The values of $\mu$ and $\mu_{1}$ are given by equations (14) which in the case of two parallel wires reduce to the following:

$$
\begin{aligned}
& -\mu^{2}=a+b=-p^{2}(L+M)\left(C+C_{12}\right)+i p R\left(C+C_{12}\right) \\
& -\mu_{1}^{2}=a-b=-p^{2}(L-M)\left(C-C_{12}\right)+i p R\left(C-C_{12}\right)
\end{aligned}
$$

$\mu$ and $\mu_{1}$ are of course complex quantities, putting

$$
\mu=\alpha+i \beta \quad \mu_{1}=\alpha_{1}+\imath \beta_{1}
$$

and solving for $\alpha, \beta, \alpha_{1}$ and $\beta_{1}$ we get

$$
\begin{aligned}
\alpha & =\sqrt{\frac{1}{2} p\left(C+C_{12}\right)\left[\sqrt{p^{2}(L+M)^{2}+R^{2}+p(L+M)}\right]} \\
\beta & \left.=\sqrt{\frac{1}{2} p\left(C+C_{12}\right)\left[\sqrt{p^{2}(L+M)^{2}+R^{2}}-p(L+M)\right.}\right] \\
\alpha_{1} & =\sqrt{\frac{1}{2} p\left(C-C_{12}\right)\left[\sqrt{p^{2}(L-M)^{2}+R^{2}}+p(\overline{L-M)}]\right.} \\
\beta & =\sqrt{\frac{1}{2} p\left(C-C_{12}\right)\left[\sqrt{p^{2}(L-M)^{2}+R^{2}-p(L-M)}\right]}
\end{aligned}
$$


It remains now to separate the values of $x$ and $y$ into the real and imaginary parts, for the actual currents in the lines will be the real parts of the values of $x$ and $y$ as given by equation (17). Now

$$
\begin{gathered}
\frac{i p E e^{i p t}}{\mu \sin \mu l}=\frac{\imath p E(\cos p t+i \sin p t)}{(\alpha+\imath \beta) \sin (\alpha+\imath \beta) l}= \\
\frac{2(\alpha-\imath \beta)\left(\sin \alpha l\left(e^{\beta l}+e^{-\beta l}\right)-i \cos \alpha l\left(e^{\beta l}-e^{-\beta l}\right)\right) E \imath p(\cos p t+\imath \sin p t)}{\left(\alpha^{2}+\beta^{2}\right)\left[\sin ^{2} \alpha l\left(e^{2 \beta l}+2+e^{-2 \beta l}\right)+\cos ^{2} \alpha l\left(e^{2 \beta l}-2+e^{-2 \beta l}\right]\right.}
\end{gathered}
$$

the real part of which is

$$
\begin{gathered}
2 E_{p}\left[\left\{\beta \cos \alpha l\left(e^{\beta l}-e_{--\beta l}^{-\beta l}-\alpha \sin \alpha l\left(e^{\beta l}+e^{-\beta l}\right)\right\} \sin p t\right.\right. \\
\left.+\left\{\beta \sin \alpha l\left(e^{\beta l}+e^{-\beta l}\right)+\alpha \cos \alpha l\left(e^{\beta l}-e^{-\beta l}\right)\right\} \cos p t\right] \\
\left(\alpha^{2}+\beta^{2}\right)\left(e^{2 \beta l}+e^{-2 \beta l}-2 \cos 2 \alpha l\right) \\
=\frac{2 E p \sin \left(p^{t} \phi\right)}{\sqrt{ }\left(\alpha^{2}+\beta^{2}\right)(2 \cos \bar{h} 2 \beta l-2 \cos 2 \alpha \bar{l})}
\end{gathered}
$$

Where $\tan \phi=$

$$
\frac{\beta \sin \alpha l\left(e^{\beta l}+e^{-\beta l}\right)+\alpha \cos \alpha l\left(e^{\beta l}-e^{-\beta l}\right)}{\bar{\beta} \cos \alpha} \frac{l\left(e^{\beta l}-e^{-\beta l}\right)-\alpha \sin \alpha l\left(e^{\beta l}+e^{-\beta l}\right)}{l}
$$

When $\beta l$ is a very small quantity, then approximately

$$
\tan \phi=-\frac{\beta}{\alpha}
$$

When $\beta l$ is very large, then the above equation will be approximately

$$
\tan \phi=\frac{\beta \sin \alpha l+\alpha \cos \alpha l}{\beta \cos \alpha l-\alpha \sin \alpha l}
$$

At the end of the line, say when $s=l$, the values of the currents will therefore be given by the following equations:

$$
\begin{aligned}
& -x_{l}=E p\left\{\frac{\left(C_{1}+C_{12}\right) \sin (p t+\phi)}{\sqrt{\left(\alpha^{2}+\beta^{2}\right)(2 \cos h 2 \beta l-2 \cos 2 \alpha l)}}\right. \\
& \left.+\frac{\left(C_{1}-C_{12}\right) \sin \left(p t+\phi_{1}\right)}{\sqrt{\left(\alpha_{1}^{2}+\beta_{1}^{2}\right)\left(2 \cos h 2 \beta_{1} l-2 \cos 2 \alpha_{1} l\right)}}\right\} \\
& -y_{l}=E p\left\{\frac{\left(C_{1}+C_{12}\right) \sin (p t+\phi)}{\sqrt{\left(\alpha^{2}+\beta^{2}\right)(2 \cos h 2 \beta l-2 \cos 2 \alpha l)}}\right. \\
& \left.-\frac{\left(C_{1}-C_{12}\right) \sin \left(p t+\phi_{1}\right)}{\sqrt{\left(\alpha_{1}{ }^{2}+\beta_{1}{ }^{2}\right)\left(2 \cos h 2 \beta_{1} l-2 \cos 2 \alpha_{1} l\right)}}\right\}
\end{aligned}
$$


The second equation of (23) represents the current at one end of the line $T_{2}$ due to the electromagnetic and electrostatic induction. We may put equation (23) in a more convenient form, thus:

$$
y_{l}=E_{p} \sqrt{A^{2}+B^{2}-2} \overline{A B \cos \left(\phi-{ }_{1} \phi\right)} \sin (p t+\phi)
$$

Where

$$
\begin{gathered}
A=\frac{C-C_{12}}{\left.\sqrt{\left(\alpha_{1}{ }^{2}+\beta_{1}{ }^{2}\right)\left(2 \cos h 2 \beta_{1} l-2 \cos 2 \alpha_{1} l\right.}\right)} \\
B=\frac{C+C_{\mathrm{i} 2}}{\sqrt{\left(\alpha^{2}+\beta^{2}\right)(2 \cos h 2 \beta l-2 \cos 2 \alpha l)}} \\
\quad \tan \phi=\frac{A \cos \phi_{1}-B \cos \phi}{A \sin \phi_{1}-B \sin \phi}
\end{gathered}
$$

The maximum value of the current will evidently be,

$$
E_{p} \sqrt{A^{2}+B^{2}-2 A B \cos \left(\phi-\phi_{1}\right)} \text {. }
$$

Now to find the ratio of the electrostatic to the electromagnetic induction, we calculate the value of $y_{l}$ as given by equation (24) first on the supposition that $M=0$; that is, that the whole effect is purely electrostatic, and then calculate the value of $y_{l}$ on putting $C_{12}=0$; that is, assuming that the effect is purely electromagnetic. We can thus obtain an estimate of the relative importance of the two forms of induction in causing disturbances in parallel lines. The values of the various electrical coefficients which enter into the calculations were determined from formulas given by $\mathrm{Mr}$. Heaviside* in his collected papers, which are as follows:

$$
\begin{gathered}
L=\frac{1}{2}+2 \log \frac{2 h}{r} \\
M=\log \frac{d^{2}+\left(h_{1}+h_{2}\right)^{2}}{d^{2}+\left(h_{1}-h_{2}\right)^{2}} \\
C_{1}=\frac{2 \log \frac{2 h}{r}}{\left(2 \log \frac{2 h}{r}\right)^{2}-\left(\log \frac{d^{2}+4 h^{2}}{d^{2}}\right)^{2}} \\
-C_{12}=\frac{\log \frac{d^{2}+4 h^{2}}{d^{2}}}{\left(2 \log \frac{2 h}{r}\right)^{2}-\left(\log \frac{d^{2}+4 h^{2}}{d^{2}}\right)^{2}}
\end{gathered}
$$

*O. Heaviside collected papers, Vol. 1, pp. 44 and 101. 
These formulas apply to the case of parallel suspended wires where $h$ is the height above the ground, $d$ is the distance between the wires, and $r$ is the radius of the wire.

Let us now consider an example; suppose the two wires are $1.25 \mathrm{~cm}$. apart, and at about $1000 \mathrm{~cm}$. above the ground let the radius of each wire be $0.1 \mathrm{~cm}$., then we find

$$
\begin{aligned}
L . & =0.00203 \text { henry per kilometre. } \\
M & =0.00147 \text { " “ } \\
C & =0.0118 \mathrm{mf} . \quad \text { “ } \\
-C_{12} & =0.0088 \mathrm{mf} .
\end{aligned}
$$

$R=3$ ohms per kilometre approximately.

Using these values for the constants of the lines, I have calculated the maximum of the current $y_{l}$, as given by equation(24),

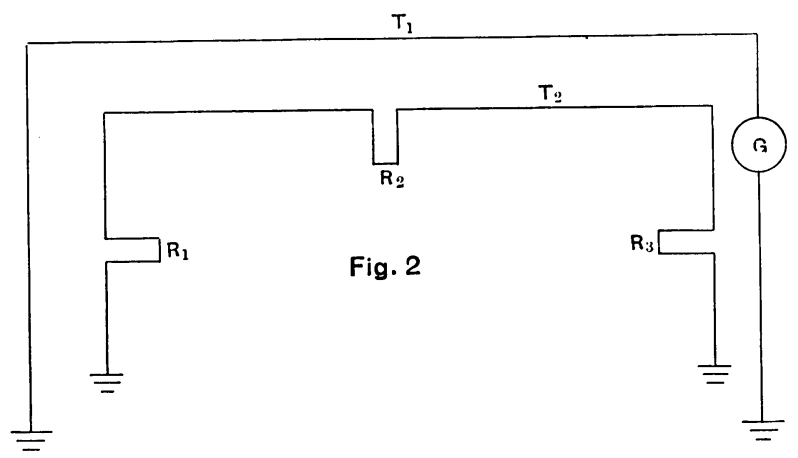

first assuming that we have only electrostatic induction;

\begin{tabular}{|c|c|}
\hline$l$ & e. $s$. \\
\hline $0.1 \mathrm{~km}$. & 0.02 \\
\hline $100 \mathrm{~km}$ & 0.44 \\
\hline $1000 \mathrm{~km}$. & 1.6 \\
\hline
\end{tabular}
that is, $M=0$, and second assuming that we have only electromagnetic induction; that is, $C_{12}=0$. The ratios of the two forms of induction for various lengths of lines given below:

From this short table it will be seen that for the particular case under consideration, the electromagnetic induction is far larger than the electrostatic; but as the length increases the 
electrostatic is gaining more rapidly than the electromagnetic, and thus at $1000 \mathrm{~km}$., the electrostatic has overpowered the electromagnetic. In obtaining the above results we have assumed that our line does not have any telephone receiver in it, which is of course not the important case. In the case of a long line, however, say $100 \mathrm{~km}$. or more, where the inductance of the line is large, compared with the inductance of the receiver; the introduction of a receiver will not modify the result to any great extent, but in the case of a short line, the introduction of one or more receivers may affect the results to a very great extent. Let us consider for example one of $\mathrm{Mr}$. Carty's experiments.

There are two lines of about $0.1 \mathrm{~km}$. long stretched side by side at a distance of about $1 \mathrm{~cm}$. and there are three telephone receivers in the second line, the complete solution of this problem is not of course so simple, yet as an approximation we may consider the introduction of the telephone receivers in such a short line as a distributed inductance and resistance. If the inductance of each receiver is 0.05 henry and its resistance is 50 ohms, then for such a short line, the inductance per unit length will be $L=1.5$ henry and $R=1500$ ohms. Assuming these to be the constants of our line we find, on calculating as in the previous cases, that the current due to electromagnetic induction is practically zero, and it is only the current due to electrostatic induction that has any appreciable value, and this is what Mr. Carty obtained experimentally. To infer from this that electromagnetic induction is negligible in all cases is however, certainly incorrect. In the case of a long line, say $500 \mathrm{~km}$., the induction and resistance of the telephone receivers will not modify to any great extent the constants of the line, and in that case the electromagnetic induction is just as important as the electrostatic induction. Which is the more important depends a great deal on the length of the lines, their height above the ground, and their distance apart. By varying any one of the above factors we shall vary the ratio of the electromagnetic to the electrostatic induction. 